\title{
ON CONVEX AND STARLIKE UNIVALENT FUNCTIONS
}

\author{
R.K. Pandey and G.P. Bhargava
}

In this paper we obtain some classical results by using the general integral operators which transform Jakubowski's class $K(m, M)$ into itself and $K(\mu) \times S(m, M)$ into $K(\mu)$. Our results generalize some recent known results due to Causey and Reade, Patil and Thakare.

\section{Introduction}

Let $S$ denote the family of functions $f(z)=z+\sum_{n=2}^{\infty} a_{n} z^{n}$ which are regular and univalent in the unit disc $D=\{z:|z|<1\}$ with the normalization $f(0)=0=f^{\prime}(0)-1$. Let $K$ and $S^{*}$ be the subfamily of $S$ whose members map $D$ onto a domain which are respectively convex and starlike with respect to origin. Robertson [4] defined the convex and starlike functions of order $\mu$ for functions $f \in S$.

A function $f$ of $S$ belongs to the class $K(\mu)$, convex functions of order $\mu, 0 \leq \mu<1$, if

$$
\operatorname{Re}\left\{1+z \frac{f^{\prime \prime}(z)}{f^{\prime}(z)}\right\}>\mu, \quad z \in D .
$$

A function $f$ of $S$ belongs to the class $S^{*}(\mu)$, starlike functions of order $\mu, 0 \leq \mu<1$, if

Received 25 July 1983.

Copyright Clearance Centre, Inc. Serial-fee code: 0004-9727/83 $\$ A 2.00+0.00$. 


$$
\operatorname{Re}\left\{z \frac{f^{\prime}(z)}{f(z)}\right\}>\mu, \quad z \in D
$$

Jakubowski [2] defined the classes $K(m, M)$ and $S(m, M)$ of functions $f \in S$.

A function $f$ of $S$ belongs to the class $K(m, M)$ if the following condition is satisfied:

$$
\left|1+\frac{z f^{\prime \prime}(z)}{f^{\prime}(z)}-m\right|<M, \quad z \in D, \quad(m, M) \in E .
$$

A function $f$ of $S$ belongs to the class $S(m, M)$ if the following condition is satisfied:

$$
\left|z \frac{f^{\prime}(z)}{f(z)}-m\right|<M, \quad z \in D, \quad(m, M) \in E
$$

where

$$
E=\{(m, M):|m-1|<M \leq m\} .
$$

Evidently

$$
K(m, M) \subset K(m-M) \subset K(0) \subset S
$$

and

$$
S(m, M) \subset S^{*}(m-M) \subset S^{*}(0) \subset S .
$$

Recently Patil and Thakare [3] established the following result.

THEOREM A. Let $\gamma$ be a real number and $f \in K(\mu)$; then the fronction $F$ defined by

$$
F(z)=\int_{0}^{z}\left\{f^{\prime}(u)\right\}^{\gamma} d u
$$

belongs to $K(n)$ for $0 \leq \gamma \leq(1-n) /(1-\mu)$.

In 1982, Causey and Reade [1] established the following result.

THEOREM B. Let $\alpha, \beta$ be real numbers and $f, g \in K$; then the function $F$ defined by

$$
F(z)=\int_{0}^{z}\left\{f^{\prime}(u)\right\}^{\alpha}\left\{\frac{g(u)}{u}\right\}^{\beta} d u
$$

belongs to $K$ only for those $(\alpha, \beta)$ in the closed convex hull of the points $(0,0),(1,0)$ and $(0,2)$. 
In this paper we study the integral operators of the form (1.8) and (1.9) which transform $K(m, M)$ into itself and $K(\mu) \times S(m, M)$ into $K(\mu)$ where $\beta$ and $\gamma$ are complex numbers. Powers in (1.8) and (1.9) are principal ones. Our results generalize the known results of Patil and Thakare [3], Causey and Reade [1].

\section{Preliminary lemmas}

LEMMA. If $f \in S$ then $f$ is in $K(m, M)$ if and only if there exists a regular function $\omega(z)$ in $D$, satisfying $\omega(0)=0$ and $|w(z)|<1$ for $z$ in $D$, such that

$$
I+\frac{z f^{\prime \prime}(z)}{f^{\prime}(z)}=\frac{1+a w(z)}{1-b w(z)}, \quad z \in D,
$$

where

$$
a=\frac{M^{2}-m^{2}+m}{M}, \quad b=\frac{m-1}{M} \text { and }(m, M) \in E
$$

Proof. To prove our lemma we require the following result.

A general bilinear transformation which maps the circular disc $|z| \leq \rho$ into the circular disc $\left|w_{1}\right| \leq \rho^{\prime}$ can be put in the form

$$
w_{1}=\rho \rho^{\prime} e^{i \lambda} \frac{z-\alpha}{\bar{\alpha} z-\rho^{2}}, \quad(|\alpha|<\rho) .
$$

Since $f \in K(m, M),\left|1+z\left(f^{\prime \prime}(z) / f^{\prime}(z)\right)-m\right|<M$. Let us take

$$
p(z)=1+z \frac{f^{\prime \prime}(z)}{f^{\prime}(z)}-m \text { so that }|p(z)|<M ;
$$

from (2.3) we have

$$
p(z)=\rho \rho^{\prime} e^{i \lambda} \frac{w_{1}(z)-\alpha}{\bar{\alpha} w_{1}(z)-\rho^{2}}=M e^{i \lambda} \frac{w_{1}(z)-\alpha}{\bar{\alpha} w_{1}(z)-1}, \rho=1, \rho^{\prime}=M .
$$

From (2.4) and (2.5) we get

$$
p(0)=(1-m)=M e^{i \lambda}(\alpha) \text { or } \alpha=((1-m) / M) e^{-i \lambda},|\alpha|<1 .
$$

Substituting the value of $\alpha$ in (2.5) we have

$$
\left\{1+z \frac{f^{\prime \prime}(z)}{f^{\prime}(z)}\right\}=m+\frac{M e^{i \lambda_{w_{1}}(z)-((1-m) / M) e^{i \lambda}}}{((1-m) / M) e^{i \lambda_{w_{1}}(z)-1}}
$$


Rearranging (2.6) by using $w(z)=-e^{i \lambda_{w_{1}}(z),} w(0)=0,|w(z)|<1$ and (2.2), we get $(2.1)$.

Conversely suppose that $f(z)$ satisfies (2.1). Then

$$
1+z \frac{f^{\prime \prime}(z)}{f^{\prime}(z)}-m=M \frac{\{(1-m) / M\}+w(z)}{1+\{(1-m) / M\} w(z)}=M h(z)
$$

say. Since $|(1-m) / M|<1$, the function $h$ given by

$$
h(z)=\frac{\{(1-m) / M\}+w(z)}{1+\{(1-m) / M\} w(z)}
$$

satisfies $|h(z)|<1$. Hence from (2.7) it follows now that the condition (2.1) is satisfied. This completes the proof of the lemma.

REMARK. Let us choose $m=N(1-\mu)+\mu$ and $M=N(1-\mu)$ where $N \geq 1$ and $0 \leq \mu<1$. Then $|m-1|<M \leq m, a=\mu / N+(1-2 \mu)$ 'and $b=1-1 / N$ also the condition $\left|1+z\left(f^{\prime \prime}(z) / f^{\prime}(z)\right)-m\right|<M$ can be written as

$$
\left|\frac{1+z\left(f^{\prime \prime}(z) / f^{\prime}(z)\right)-\mu}{1-\mu}-N\right|<N, \quad z \in D \text {. }
$$

Now, as $N \rightarrow \infty, a \rightarrow(1-2 \mu)$ and $b \rightarrow 1$, the condition (2.8) reduces to $\operatorname{Re}\left\{1+z\left(f^{\prime \prime}(z) / f^{\prime}(z)\right)\right\}>\mu, z \in D$. In this case the relation (2.1) becomes

$$
1+z \frac{f^{\prime \prime}(z)}{f^{\prime}(z)}=\frac{1+(1-2 \mu) \omega(z)}{1-\omega(z)}, \quad z \in D
$$

which is a necessary and sufficient condition for $f$ to be in $K(\mu)$.

\section{Class preserving integral operator for $K(m, M)$}

THEOREM. Let $\gamma$ be a complex number and $f \in K(m, M)$; then the function $F$ defined by

$$
F(z)=\int_{0}^{z}\left\{f^{\prime}(u)\right\}^{\gamma} d u
$$

belongs to $K(m, M)$ for $0 \leq|\gamma| \leq \frac{1}{2}(1-b)$.

Proof. Let us choose a function $w$ such that

$$
1+z \frac{F^{\prime \prime}(z)}{F^{\prime}(z)}=\frac{1+a w(z)}{1-b w(z)}
$$

where $w(0)=0$ and $w$ is either regular or meromorphic in $D$. From 
(3.1) we have

$$
F^{\prime}(z)=\left\{f^{\prime}(z)\right\}^{\gamma} .
$$

Differentiating logarithmically (3.3) with respect to $z$ and using (3.2) we get

$$
\frac{z f^{\prime \prime}(z)}{f^{\prime}(z)}=\frac{(a+b) w(z)}{\gamma(1-b w(z))}
$$

or

$$
1+z \frac{f^{\prime \prime}(z)}{f^{\prime}(z)}-m=\frac{(1-m) \gamma+[a+b\{1-(1-m) \gamma\}] w(z)}{\gamma\{1-b w(z)\}} \text {. }
$$

Let $z_{1}$ with $\left|z_{1}\right|=r_{0}$ be the nearest pole of $\omega(z)$ in $D$. Hence $w(z)$ is regular in $|z|<r_{0}<1$. Thus for $|z| p<r_{0}$ there is a point $z_{0}$ for which

$$
1+z_{0} \frac{f^{\prime \prime}\left(z_{0}\right)}{f^{\prime}\left(z_{0}\right)}-m=\frac{(1-m) \gamma+[a+b\{1-(1-m) \gamma\}] w\left(z_{0}\right)}{\gamma\left\{1-b w\left(z_{0}\right)\right\}} \equiv \frac{N\left(z_{0}\right)}{D\left(z_{0}\right\}}
$$

where

$$
\begin{aligned}
& N\left(z_{0}\right)=(1-m) \gamma+[a+b\{1-(1-m) \gamma\}] \omega\left(z_{0}\right), \\
& D\left(z_{0}\right)=\gamma\left(1-b w\left(z_{0}\right)\right) .
\end{aligned}
$$

Now suppose that it were possible to have $M(r, w)=\max _{|z|=r} w\left(z_{0}\right)=1$ for some $r<r_{0}<1$. At the point $z_{0}$ where this occurred we would have $\left|w\left(z_{0}\right)\right|=1$.

CASE 1. When $\operatorname{Re}(\gamma) \geq 0, \operatorname{Im}(\gamma) \geq 0$ and $\operatorname{Re}(\gamma) \geq 0, \operatorname{Im}(\gamma)<0$, (3.9) $\left|N\left(z_{0}\right)\right|^{2}=(a+b)^{2}+\left(1+b^{2}\right) M^{2}|\gamma|^{2}-2(a+b) M \operatorname{Re}\left\{\gamma \omega\left(z_{0}\right)\right\}$

$$
-2 b M^{2}|\gamma|^{2} \operatorname{Re}\left(w\left(z_{0}\right)\right\}+2 b M(a+b) \operatorname{Re}(\gamma),
$$

(3.10) $\left|D\left(z_{0}\right)\right|^{2}=\left(1+b^{2}\right)|\gamma|^{2}-2 b|\gamma|^{2} \operatorname{Re}\left(w\left(z_{0}\right)\right)$.

CASE 2. When $\operatorname{Re}(\gamma)<0, \operatorname{Im}(\gamma)<0$ and $\operatorname{Re}(\gamma)<0, \operatorname{Im}(\gamma) \geq 0$, 


$$
\begin{aligned}
\left|N\left(z_{0}\right)\right|^{2}=(a+b)^{2}+\left(1+b^{2}\right) M^{2}|\gamma|^{2} & +2(a+b) M \operatorname{Re}\left\{\gamma \omega\left(z_{0}\right)\right\} \\
& -2 b M^{2}|\gamma|^{2} \operatorname{Re}\left(\omega\left(z_{0}\right)\right)-2 b M(a+b) \operatorname{Re}(\gamma)
\end{aligned}
$$

and

$$
\left|D\left(z_{0}\right)\right|^{2}=\left(1+b^{2}\right)|\gamma|^{2}-2 b|\gamma|^{2} \operatorname{Re}\left(w\left(z_{0}\right)\right)
$$

Now for each case

$$
\begin{aligned}
\left|N\left(z_{0}\right)\right|^{2}-M^{2}\left|D\left(z_{0}\right)\right|^{2} & \geq(a+b)^{2}-2 M(a+b)(1+b)|\gamma| \\
& \geq 0 \text { for }|\gamma| \leq \frac{1}{2}(1-b) .
\end{aligned}
$$

Thus from (3.6) it follows that

$$
\left|1+z_{0} \frac{f^{\prime \prime}\left(z_{0}\right)}{f^{\prime}\left(z_{0}\right)}-m\right|>M \text { for }|\gamma| \leq \frac{1}{2}(1-b) .
$$

But this is contrary to the fact that $f \in K(m, M)$. So we cannot have $M(r, w)=1$. Thus $|w(z)| \neq 1$ in $|z|<x_{0}$. Since $|w(0)|=0$, $|w(z)|$ is continuous and $|w(z)| \neq 1$ in $|z|<r_{0}, w(z)$ cannot have a pole at $\left|z_{1}\right|=r_{0}$. Therefore $w(z)$ is regular and $|w(z)|<1$ for $z$ in $D$.

Hence $F \in K(m, M)$.

4. Integral operator that maps $K(\mu) \times S(m, M)$ into $K(\mu)$

THEOREM. Let $\alpha$ be a non zero positive real number and $\beta$ be $a$ complex number such that $0 \leq|\beta| \leq-(\alpha-1) / 2 M,(m, M) \in E$.

Let $f \in K(\mu)$ and $g \in S(m, M)$; then the fronction $F$ defined by

$$
F(z)=\int_{0}^{z}\left\{f^{\prime}(u)\right\}^{\alpha}\left\{\frac{g(u)}{u}\right\}^{\beta} d u
$$

belongs to $K(\mu)$.

Proof. Let us choose a function $w$ such that

$$
1+z \frac{f^{\prime \prime}(z)}{f^{\prime}(z)}=\frac{1+(2 \mu-1) w(z)}{1+w(z)}
$$


where $w(0)=0$ and $w$ is either regular or meromorphic in $D$.

Differentiating (4.1) with respect to $z$ we have

$$
F^{\prime}(z)=\left\{f^{\prime}(z)\right\}^{\alpha}\left\{\frac{g(z)}{z}\right\}^{\beta} \text {. }
$$

Differentiating logarithmically (4.3) with respect to $z$ and using (4.2) we have

(4.4) $\left\{1+z \frac{f^{\prime \prime}(z)}{f^{\prime}(z)}\right\}=\frac{\alpha+(1-m) B}{\alpha}-\frac{\beta}{\alpha}\left\{z \frac{g^{\prime}(z)}{g(z)}-m\right\}+\frac{2(\mu-1)}{\alpha} \frac{w(z)}{1+w(z)}$.

Let $z_{1}$ with $\left|z_{1}\right|=r_{0}$ be the nearest pole of $w(z)$ in $D$. Hence $w(z)$ is reguiar in $|z|<r_{0}<1$. Thus for $|z| \leq r<r_{0}$ there is a point $z_{0}$ for which

(4.5) $\left\{1+z_{0} \frac{f^{\prime \prime}\left(z_{0}\right)}{f^{\prime}\left(z_{0}\right)}\right\}=\frac{\alpha-(m-1) \beta}{\alpha}-\frac{\beta}{\alpha}\left\{z_{0} \frac{g^{\prime}\left(z_{0}\right)}{g\left(z_{0}\right)}-m\right\}+\frac{2(\mu-1)}{\alpha} \frac{w\left(z_{0}\right)}{1+w\left(z_{0}\right)}$,

or

(4.6) $\operatorname{Re}\left\{1+z_{0} \frac{f^{\prime \prime}\left(z_{0}\right)}{f^{\prime}\left(z_{0}\right)}\right\} \leq \frac{\alpha+(m-1)|\beta|}{\alpha}+\frac{|\beta|}{\alpha}\left|z_{0} \frac{g^{\prime}\left(z_{0}\right)}{g\left(z_{0}\right)}-m\right|$

$$
+\frac{2(\mu-1)}{\alpha} \frac{\operatorname{Re} w\left(z_{0}\right)+\left|w\left(z_{0}\right)\right|^{2}}{1+2 \operatorname{Re} w\left(z_{0}\right)+\left|w\left(z_{0}\right)\right|^{2}}
$$

Now suppose that it were possible to have $M(r, w)=\max _{|a|=r}\left|w\left(z_{0}\right)\right|=1$ for some $r<r_{0}<1$. At the point $z_{0}$ where this occurred we would have

$$
\begin{aligned}
\operatorname{Re}\left\{1+z_{0} \frac{f^{\prime \prime}\left(z_{0}\right)}{\left.f^{\prime}\left(z_{0}\right)\right\}}\right. & <\frac{\alpha+M|\beta|}{\alpha}+\frac{|\beta|}{\alpha} M+\frac{(\mu-1)}{\alpha} \\
& \leq \mu \text { for }|\beta| \leq-\frac{(\alpha-1)}{2 M} .
\end{aligned}
$$

But this is contrary to the fact that $f \in K(\mu)$. So we cannot have $M(r, w)=1$. Thus $|w(z)| \neq 1$ in $|z|<r_{0}$. Since $w(0)=0,|w(z)|$ is continuous in $|z|<r_{0}$ and $|w(z)| \neq 1$ where $w(z)$ cannot have a pole at $\left|z_{1}\right|=r_{0}$. Therefore $|w(z)|<1$ and $w(z)$ is regular in $D$. 
Hence from (4.2) it follows that $F \in K(\mu)$.

APPLICATIONS. By using the same techniques, we can also study the following types of integral operators of the forms

$$
\begin{aligned}
\text { (i) } F(z) & =\int_{0}^{z}\{f(u) / u\}^{\beta} d u, \\
\text { (ii) } F(z) & =\int_{0}^{z}\left\{f^{\prime}(u)\right\}^{\alpha}\left\{g^{\prime}(u)\right\}^{\beta} d u \text {, and } \\
\text { (iii) } F(z) & =\int_{0}^{z}\{f(u) / u\}^{\alpha}\{g(u) / u\}^{\beta} d u,
\end{aligned}
$$

which transform $S(m, M)$ into $K(m, M), K(\mu) \times K(m, M)$ into $K(\mu)$ and $S^{*}(\mu) \times S(m, M)$ into $K(\mu)$ respectively where $\alpha$ is a real number and $\beta$ is a complex number.

\section{References}

[1] W.M. Causey and M.O. Reade, "On the univalence of functions defined by certain integral transforms", J. Math. Anal. Appl. 89 (1982), 28-39.

[2] 2.J. Jakubowski, "On the coefficients of starlike functions of some classes", Ann. Polon. Math. 26 (1972), 305-313.

[3] D.A. Patil and N.K. Thakare, "On univalence of certain integrals", Indian J. Pure App Z. Math. 11 (1980), 1626-1642.

[4] M.S. Robertson, "On the theory of univalent functions", Ann. of Math. (2) $37(1936), 374-408$.

[5] S.L. Shukla and V. Kumar, "Jakubowski starlike integral operators", $J$. Austral. Math. Soc. Ser. A (to appear).

Department of Mathematics,

PPN Coll lege,

Kanpur - 208001 ,

India. 